\title{
QUANTITATIVE ESTIMATES FOR SIMPLE ZEROS OF $L$-FUNCTIONS
}

\author{
ANDREW R. BOOKER, MICAH B. MILINOVICH, AND NATHAN NG
}

AbstraCt. We generalize a method of Conrey and Ghosh CG88 to prove quantitative estimates for simple zeros of modular form $L$-functions of arbitrary conductor.

\section{INTRODUCTION}

Let $f \in S_{k}\left(\Gamma_{1}(N)\right)$ be a classical holomorphic modular form of weight $k$ and level $N$. Assume that $f$ is primitive, meaning that it is a normalized Hecke eigenform in the new subspace. Then it has a Fourier expansion of the shape

$$
f(z)=\sum_{n=1}^{\infty} \lambda_{f}(n) n^{\frac{k-1}{2}} e^{2 \pi i n z},
$$

where the $\lambda_{f}(n)$ are multiplicative and satisfy the Ramanujan bound $\left|\lambda_{f}(n)\right| \leq d(n)$. Let $\Lambda_{f}(s)=\Gamma_{\mathbb{C}}\left(s+\frac{k-1}{2}\right) L_{f}(s)$ denote the complete $L$-function of $f$, with analytic normalization, where

$$
\Gamma_{\mathbb{C}}(s)=2(2 \pi)^{-s} \Gamma(s) \quad \text { and } \quad L_{f}(s)=\sum_{n=1}^{\infty} \frac{\lambda_{f}(n)}{n^{s}},
$$

and let

$$
N_{f}^{s}(T)=\#\left\{\rho \in \mathbb{C}: \Lambda_{f}(\rho)=0, \Lambda_{f}^{\prime}(\rho) \neq 0,|\Im(\rho)| \leq T\right\}
$$

be the number of simple zeros of $\Lambda_{f}(s)$ with imaginary part in $[-T, T]$.

In [MN14, the second and third authors showed that if $\Lambda_{f}(s)$ satisfies the Generalized Riemann Hypothesis, then

$$
N_{f}^{s}(T) \geq T(\log T)^{-\varepsilon}
$$

for any fixed $\varepsilon>0$ and all sufficiently large $T>0$. Unconditionally, when $N=1$ and $k=12$, Conrey and Ghosh CG88] showed that

$$
\forall \varepsilon>0, \exists T \geq \varepsilon^{-1} \text { such that } N_{f}^{s}(T) \geq T^{\frac{1}{6}-\varepsilon} .
$$

Moreover, their proof works more generally for $N=1$ and arbitrary $k$, provided that $N_{f}^{s}(T)$ is not identically 0. In light of the first author's result [Boo16] that $N_{f}^{s}(T) \rightarrow \infty$ as $T \rightarrow \infty$, (1.1) holds for all primitive $f$ of conductor 1 .

In this paper we aim to prove similar unconditional quantitative estimates of simple zeros for primitive forms of arbitrary conductor $N$. However, we encounter some obstacles that are reminiscent of the well-known difficulty of extending Hecke's converse theorem to arbitrary conductor, and are not present for $N=1$. Taking inspiration from Weil's generalization [Wei67] of Hecke's converse theorem, we consider character twists. For

2010 Mathematics Subject Classification. Primary 11F66, 11F11, $11 \mathrm{M} 41$.

Research of the first author was supported by EPSRC Grant EP/K034383/1. Research of the second author was supported by the NSA Young Investigator Grants H98230-15-1-0231 and H98230-16-1-0311. Research of the third author was supported by NSERC Discovery Grant (RGPIN2015-05972). No data were created in the course of this study. 
a Dirichlet character $\chi(\bmod q)$, let $f \otimes \chi$ denote the unique primitive form such that $\lambda_{f \otimes \chi}(n)=\lambda_{f}(n) \chi(n)$ for all $n$ coprime to $q$.

Theorem 1.1. Let $f \in S_{k}\left(\Gamma_{1}(N)\right)$ be a primitive form. Then there is a Dirichlet character $\chi$ such that (1.1) holds with $f \otimes \chi$ in place of $f$.

Next, for odd conductors we obtain a weaker but unconditional quantitative estimate for $N_{f}^{s}(T)$, without the twist. Moreover, we show that there is a sort of "DeuringHeilbronn phenomenon" at play, so that if $N_{f}^{s}(T)$ is unexpectedly small then we can substantially improve our result for $N_{f \otimes \chi}^{s}(T)$.

Theorem 1.2. Let $f \in S_{k}\left(\Gamma_{1}(N)\right)$ be a primitive form of odd conductor. Then

$$
\forall \varepsilon>0, \exists T \geq \varepsilon^{-1} \text { such that } N_{f}^{s}(T) \geq \begin{cases}\exp \left((\log T)^{\frac{1}{3}-\varepsilon}\right) & \text { if } k=1 \text { or } f \text { is a } C M \text { form, } \\ \log \log \log T & \text { otherwise. }\end{cases}
$$

Further, if $N_{f}^{s}(T) \ll 1+T^{\varepsilon}$ for every $\varepsilon>0$, then

(i) there is a Dirichlet character $\chi$ such that, $\forall \varepsilon>0, \exists T \geq \varepsilon^{-1}$ such that $\Lambda_{f \otimes \chi}(s)$ has at least $T^{\frac{1}{2}-\varepsilon}$ simple zeros with real part $\frac{1}{2}$ and imaginary part in $[-T, T]$;

(ii) $\Lambda_{f}(s)$ has simple zeros with real part arbitrarily close to 1 .

Remarks.

(1) The exponent $\frac{1}{6}$ in (1.1) is related to the best known subconvexity estimate for modular form $L$-functions in the $t$ aspect; it can be replaced by any $\delta>0$ such that $L_{f}\left(\frac{1}{2}+i t\right) \ll_{f, \varepsilon}(1+|t|)^{\frac{1}{2}-\delta+\varepsilon}$ holds for all primitive forms $f$ and all $\varepsilon>0$. In BMN19] we showed that $\delta=\frac{1}{6}$ is admissible. Very recent work of Munshi Mun18 improves this to $\delta=\frac{1}{6}+\frac{1}{1200}$ for forms of level 1, with a corresponding improvement to (1.1) in that case.

(2) In Theorem 1.1, one can take the conductor of $\chi$ to be 1 or a prime number bounded by a polynomial function of $N$.

(3) The proof of Theorem 1.2 makes use of the idea originating with Conrey and Ghosh [CG88 of twisting the coefficients of $L_{f}(s)$ by $(-1)^{n}$ to prevent the main terms of our estimate from cancelling out. This relies implicitly on the fact that there is no primitive Dirichlet character of conductor 2, and is the ultimate reason for our restriction to odd $N$.

(4) The improved estimate in Theorem 1.2 in the Galois and CM cases arises from Coleman's Vinogradov-type zero-free region for Hecke $L$-functions [Col90].

Acknowledgements. We thank the anonymous referee for helpful suggestions and corrections, and the Banff International Research Station for hosting us for a Research in Teams Meeting (15rit201). A significant portion of this project was completed during that week and we appreciated the excellent working conditions.

\section{DiRICHLET SERIES}

In order to establish the existence of simple zeros it is useful to study not only $L_{f}(s)$, but some related Dirichlet series and their additive twists. This is one of the central ideas in CG88. A key role is played by the series

$$
D_{f}(s)=L_{f}(s) \frac{d^{2}}{d s^{2}} \log L_{f}(s)=\sum_{n=1}^{\infty} c_{f}(n) n^{-s},
$$


which has a meromorphic continuation to $\mathbb{C}$ with poles precisely at the simple zeros of $L_{f}(s)$ (including the trivial zeros $s=\frac{1-k}{2}-n$ for $n=0,1,2, \ldots$ ).

For $\alpha \in \mathbb{Q}^{\times}$and $\chi$ a Dirichlet character, let

$$
L_{f}(s, \alpha)=\sum_{n=1}^{\infty} \lambda_{f}(n) e(\alpha n) n^{-s} \text { and } \quad L_{f}(s, \chi)=\sum_{n=1}^{\infty} \lambda_{f}(n) \chi(n) n^{-s} .
$$

Likewise, define

$$
D_{f}(s, \alpha)=\sum_{n=1}^{\infty} c_{f}(n) e(\alpha n) n^{-s} \text { and } \quad D_{f}(s, \chi)=\sum_{n=1}^{\infty} c_{f}(n) \chi(n) n^{-s} .
$$

Let $\xi$ denote the nebentypus character of $f$. Set

$$
Q(N)=\{1\} \cup\{q \text { prime }: q \nmid N\},
$$

and for each $q \in Q(N)$, define the rational functions

$$
P_{f, q}(x)= \begin{cases}1 & \text { if } q=1 \\ 1-\lambda_{f}(q) x+\xi(q) x^{2} & \text { otherwise }\end{cases}
$$

and

$$
R_{f, q}(x)= \begin{cases}0 & \text { if } q=1, \\ \frac{q \log ^{2} q}{q-1} \frac{x\left(\lambda_{f}(q)-4 \xi(q) x+\lambda_{f}(q) \xi(q) x^{2}\right)}{P_{f, q}(x)} & \text { if } q \neq 1 .\end{cases}
$$

These are such that, if

$$
\chi_{0}(n)= \begin{cases}1 & \text { if }(n, q)=1 \\ 0 & \text { otherwise }\end{cases}
$$

denotes the trivial character $\bmod q$, then

$$
L_{f}\left(s, \chi_{0}\right)=P_{f, q}\left(q^{-s}\right) L_{f}(s)
$$

and

$$
D_{f}\left(s, \chi_{0}\right)=P_{f, q}\left(q^{-s}\right) D_{f}(s)-\frac{q-1}{q} R_{f, q}\left(q^{-s}\right) L_{f}(s) .
$$

For any $a \in \mathbb{Z}$ coprime to $q$, we define

$$
\begin{aligned}
& D_{f, a, q}(s)=D_{f}\left(s, \frac{a}{q}\right)-R_{f, q}\left(q^{-s}\right) L_{f}(s)=\sum_{n=1}^{\infty} c_{f, a, q}(n) n^{-s}, \\
& D_{f, a, q}^{*}(s)=D_{f, a, q}(s)+\psi^{\prime}\left(s+\frac{k-1}{2}\right) L_{f}\left(s, \frac{a}{q}\right), \quad \text { where } \psi(s)=\frac{\Gamma^{\prime}}{\Gamma}(s)
\end{aligned}
$$

and

$$
D_{f, a, q}(s, \alpha)=\sum_{n=1}^{\infty} c_{f, a, q}(n) e(\alpha n) n^{-s} \text { for } \alpha \in \mathbb{Q}^{\times} .
$$

To each of $L_{f}, D_{f}, D_{f, a, q}, D_{f, a, q}^{*}$ and their twists, we define completed versions $\Lambda_{f}, \Delta_{f}$, $\Delta_{f, a, q}, \Delta_{f, a, q}^{*}$ obtained by multiplying by $\Gamma_{\mathbb{C}}\left(s+\frac{k-1}{2}\right)$. By the Ramanujan bound $\left|\lambda_{f}(q)\right| \leq$ 2 and [BK11, Proposition 3.1], $\Delta_{f}(s, a / q)-\Delta_{f, a, q}^{*}(s)$ is holomorphic for $\Re(s)>0$. In turn, the analytic properties of $\Delta_{f, a, q}^{*}(s)$ are described by the following proposition. 
Proposition 2.1. Let $f \in S_{k}\left(\Gamma_{0}(N), \xi\right)$ be a primitive form, $q \in Q(N)$, and $a \in \mathbb{Z}$ coprime to $q$. Then $\Delta_{f, a, q}^{*}(s)$ is a ratio of entire functions of finite order, has at most simple poles, all of which are contained in the critical strip $\{s \in \mathbb{C}: \Re(s) \in(0,1)\}$, and satisfies the functional equation

$$
\Delta_{f, a, q}^{*}(s)=\epsilon \xi(q)\left(N q^{2}\right)^{\frac{1}{2}-s} \Delta_{\bar{f},-\overline{N a}, q}^{*}(1-s),
$$

where $\bar{f} \in S_{k}\left(\Gamma_{0}(N), \bar{\xi}\right)$ is the dual of $f, \epsilon \in \mathbb{C}^{\times}$is the root number of $f$ and $\overline{N a}$ denotes a multiplicative inverse of $N a(\bmod q)$.

Proof. For $q=1$ the result follows immediately from [Boo16, (3.1)], so we may assume that $q$ is prime. Let $\chi$ be a Dirichlet character of conductor $q$. Then the complete twisted $L$-function $\Lambda_{f}(s, \chi)$ satisfies the functional equation

$$
\Lambda_{f}(s, \chi)=\epsilon \xi(q) \chi(N) \frac{\tau(\chi)^{2}}{q}\left(N q^{2}\right)^{\frac{1}{2}-s} \Lambda_{\bar{f}}(1-s, \bar{\chi}),
$$

where $\epsilon \in \mathbb{C}^{\times}$is the root number of $f$. Applying [Boo16, (3.1)] to $f \otimes \chi$, we thus have $(2.3)$

$$
\Delta_{f}(s, \chi)-\epsilon \xi(q) \chi(N) \frac{\tau(\chi)^{2}}{q}\left(N q^{2}\right)^{\frac{1}{2}-s} \Delta_{\bar{f}}(1-s, \bar{\chi})=\Lambda_{f}(s, \chi)\left(\psi^{\prime}\left(\frac{k+1}{2}-s\right)-\psi^{\prime}\left(s+\frac{k-1}{2}\right)\right) .
$$

Next, we have

$$
\Delta_{f}\left(s, \frac{a}{q}\right)=\Delta_{f}(s)-\frac{q}{q-1} \Delta_{f}\left(s, \chi_{0}\right)+\frac{1}{q-1} \sum_{\substack{(\bmod q) \\ \chi \neq \chi_{0}}} \tau(\bar{\chi}) \chi(a) \Delta_{f}(s, \chi),
$$

where $\chi_{0}$ is the trivial character $\bmod q$. Combining this with (2.1) we get

$$
\Delta_{f, a, q}(s)=\left(1-\frac{q}{q-1} P_{f, q}\left(q^{-s}\right)\right) \Delta_{f}(s)+\frac{1}{q-1} \sum_{\substack{\chi(\bmod q) \\ \chi \neq \chi_{0}}} \tau(\bar{\chi}) \chi(a) \Delta_{f}(s, \chi) .
$$

Note in particular that $\Delta_{f, a, q}(s)$ is a ratio of entire functions of finite order, and all of its poles in $\{s \in \mathbb{C}: \Re(s)>0\}$ are simple and located at simple zeros of either $\Lambda_{f}(s)$ or $\Lambda_{f}(s, \chi)$ for some $\chi \neq \chi_{0}$.

Note that $P_{f, q}$ satisfies the functional equation

$$
1-\frac{q}{q-1} P_{f, q}\left(q^{-s}\right)=\xi(q) q^{1-2 s}\left(1-\frac{q}{q-1} P_{\bar{f}, q}\left(q^{s-1}\right)\right),
$$

and thus, by [Boo16, (3.1)],

$$
\begin{aligned}
\left(1-\frac{q}{q-1} P_{f, q}\left(q^{-s}\right)\right) & \Delta_{f}(s)-\epsilon \xi(q)\left(N q^{2}\right)^{\frac{1}{2}-s}\left(1-\frac{q}{q-1} P_{\bar{f}, q}\left(q^{s-1}\right)\right) \Delta_{\bar{f}}(1-s) \\
& =\left(1-\frac{q}{q-1} P_{f, q}\left(q^{-s}\right)\right) \Lambda_{f}(s)\left(\psi^{\prime}\left(\frac{k+1}{2}-s\right)-\psi^{\prime}\left(s+\frac{k-1}{2}\right)\right) .
\end{aligned}
$$

Thus, replacing $f$ by $\bar{f}, s$ by $1-s, a$ by $-\overline{N a}$ and $\chi$ by $\bar{\chi}$ in (2.4), we get

$$
\Delta_{\bar{f},-\overline{N a}, q}(1-s)=\left(1-\frac{q}{q-1} P_{\bar{f}, q}\left(q^{s-1}\right)\right) \Delta_{\bar{f}}(1-s)+\frac{1}{q-1} \sum_{\substack{(\bmod q) \\ \chi \neq \chi_{0}}} \tau(\chi) \chi(-N a) \Delta_{\bar{f}}(1-s, \bar{\chi}) .
$$


Applying the functional equations (2.3) and (2.5), together with the relation $\tau(\chi) \tau(\bar{\chi})=$ $\chi(-1) q$, we thus have

$$
\begin{aligned}
& \Delta_{f, a, q}(s)-\epsilon \xi(q)\left(N q^{2}\right)^{\frac{1}{2}-s} \Delta_{\bar{f},-\overline{N a}, q}(1-s) \\
& =\left[\left(1-\frac{q}{q-1} P_{f, q}\left(q^{-s}\right)\right) \Lambda_{f}(s)+\frac{1}{q-1} \sum_{\substack{(\bmod q) \\
\chi \neq \chi_{0}}} \tau(\bar{\chi}) \chi(a) \Lambda_{f}(s, \chi)\right]\left(\psi^{\prime}\left(\frac{k+1}{2}-s\right)-\psi^{\prime}\left(s+\frac{k-1}{2}\right)\right) \\
& =\Lambda_{f}\left(s, \frac{a}{q}\right)\left(\psi^{\prime}\left(\frac{k+1}{2}-s\right)-\psi^{\prime}\left(s+\frac{k-1}{2}\right)\right) .
\end{aligned}
$$

Applying the classical Voronoi formula [KMV02, p. 179, (A.10)]

$$
\Lambda_{f}\left(s, \frac{a}{q}\right)=\epsilon \xi(q)\left(N q^{2}\right)^{\frac{1}{2}-s} \Lambda_{\bar{f}}\left(1-s,-\frac{\overline{N a}}{q}\right),
$$

we arrive at (2.2).

Finally, by (2.4) and the nonvanishing of automorphic $L$-functions for $\Re(s) \geq 1$ [JS77], $\Delta_{f, a, q}^{*}(s)$ is holomorphic for $\Re(s) \geq 1$. This conclusion applies to $\Delta_{\bar{f},-\overline{N a}, q}^{*}(s)$ as well, so by (2.2), all poles of $\Delta_{f, a, q}^{*}(s)$ have real part in $(0,1)$.

Fix, for the remainder of this section, a choice of $f, a, q$ as in Proposition 2.1, and $\alpha \in \mathbb{Q}^{\times}$. We define

$$
N_{f, a, q}^{s}(T)=\#\left\{\rho \in \mathbb{C}:|\Im(\rho)| \leq T, \operatorname{Res}_{s=\rho} \Delta_{f, a, q}^{*}(s) \neq 0\right\}
$$

and

$$
S_{f, a, q}(y, \alpha)=\sum_{\rho \in \mathbb{C}} \operatorname{Res}_{s=\rho} \Delta_{f, a, q}^{*}(s)(y-i \alpha)^{-\rho-\frac{k-1}{2}} \quad \text { for } y \in \mathbb{R}_{>0},
$$

where $(y-i \alpha)^{-\rho-\frac{k-1}{2}}$ is defined in terms of the principal branch of $\log (y-i \alpha)$. Our goal is to derive the following expression for the Mellin transform of $S_{f, a, q}(y, \alpha)$, up to a holomorphic function on $\{s \in \mathbb{C}: \Re(s)>0\}$ :

Proposition 2.2. Define

$$
H_{f, a, q, \alpha}(s)=\Delta_{f, a, q}(s, \alpha)-\epsilon \xi(q)(i \operatorname{sgn} \alpha)^{k}\left(N q^{2} \alpha^{2}\right)^{s-\frac{1}{2}} \Delta_{\bar{f},-\overline{N a}, q}\left(s,-\frac{1}{N q^{2} \alpha}\right)
$$

and

$$
I_{f, a, q, \alpha}(s)=\int_{0}^{|\alpha| / 4} S_{f, a, q}(y, \alpha) y^{s+\frac{k-1}{2}} \frac{d y}{y} .
$$

Then $I_{f, a, q, \alpha}(s)-H_{f, a, q, \alpha}(s)$ has analytic continuation to $\Re(s)>0$. Moreover, if

$$
\int_{0}^{|\alpha| / 4}\left|S_{f, a, q}(y, \alpha)\right| y^{\sigma+\frac{k-1}{2}} \frac{d y}{y}<\infty
$$

for some $\sigma \geq 0$, then $H_{f, a, q, \alpha}(s)$ is holomorphic for $\Re(s)>\sigma$.

The proof will be carried out in several lemmas, and involves the following auxiliary functions defined on $\mathbb{H}=\{z \in \mathbb{C}: \Im(z)>0\}$ :

$$
\begin{aligned}
& F(z)=2 \sum_{n=1}^{\infty} c_{f, a, q}(n) n^{\frac{k-1}{2}} e(n z), \quad \bar{F}(z)=2 \sum_{n=1}^{\infty} c_{\bar{f},-\overline{N a}, q}(n) n^{\frac{k-1}{2}} e(n z), \\
& A(z)=\frac{1}{2 \pi i} \int_{\Re(s)=\frac{k}{2}} \Lambda_{f}\left(s, \frac{a}{q}\right)\left(\psi^{\prime}\left(s+\frac{k-1}{2}\right)+\psi^{\prime}\left(s-\frac{k-1}{2}\right)\right)(-i z)^{-s-\frac{k-1}{2}} d s,
\end{aligned}
$$


and

$$
B(z)=\frac{1}{2 \pi i} \int_{\Re(s)=\frac{k}{2}} \Lambda_{f}\left(s, \frac{a}{q}\right) \frac{\pi^{2}}{\sin ^{2}\left(\pi\left(s+\frac{k-1}{2}\right)\right)}(-i z)^{-s-\frac{k-1}{2}} d s .
$$

We first derive the following expression for $S_{f, a, q}$.

Lemma 2.3. For $z=\alpha+i y \in \mathbb{H}$, we have

$$
S_{f, a, q}(y, \alpha)=F(z)-\frac{\epsilon \xi(q)}{(-i \sqrt{N} q z)^{k}} \bar{F}\left(-\frac{1}{N q^{2} z}\right)+A(z)-B(z) .
$$

Proof. Let $0<\varepsilon<\frac{1}{2}$. For $z \in \mathbb{H}$ we define

$I_{R}(z)=\frac{1}{2 \pi i} \int_{\Re(s)=1+\varepsilon} \Delta_{f, a, q}(s)(-i z)^{-s-\frac{k-1}{2}} d s, \quad I_{L}(z)=\frac{1}{2 \pi i} \int_{\Re(s)=-\varepsilon} \Delta_{f, a, q}(s)(-i z)^{-s-\frac{k-1}{2}} d s$.

For the remainder of the proof we let $z=\alpha+i y$.

Since $\Delta_{f, a, q}^{*}(s)$ is a ratio of entire functions of finite order with at most simple poles, by the calculus of residues we have

$$
\operatorname{Res}_{s=0} \Delta_{f, a, q}(s)(-i z)^{-s-\frac{k-1}{2}}+S_{f, a, q}(y, \alpha)=I_{R}(z)-I_{L}(z) .
$$

Note that the residue term at $s=0$ vanishes unless $k=1$. We have

$$
\begin{aligned}
I_{R}(z) & =\frac{1}{2 \pi i} \int_{\Re(s)=1+\varepsilon} \Gamma_{\mathbb{C}}\left(s+\frac{k-1}{2}\right) D_{f, a, q}(s)(-i z)^{-s-\frac{k-1}{2}} d s \\
& =2(-2 \pi i z)^{-\frac{k-1}{2}} \sum_{n=1}^{\infty} c_{f, a, q}(n) \frac{1}{2 \pi i} \int_{\Re(s)=1+\varepsilon} \Gamma\left(s+\frac{k-1}{2}\right)(-2 \pi i n z)^{-s} d s .
\end{aligned}
$$

Using the identity

$$
\frac{1}{2 \pi i} \int_{\Re(s)=1+\varepsilon} \Gamma\left(s+\frac{k-1}{2}\right) z^{-s} d s=z^{\frac{k-1}{2}} e^{-z} \quad \text { for } \Re(z)>0,
$$

it follows that

$$
I_{R}(z)=2 \sum_{n=1}^{\infty} c_{f, a, q}(n) n^{\frac{k-1}{2}} e(n z)=F(z) .
$$

By the functional equation, we have

$$
\Delta_{f, a, q}(s)=\epsilon \xi(q)\left(N q^{2}\right)^{\frac{1}{2}-s} \Delta_{\bar{f},-\overline{N a}, q}(1-s)+\Lambda_{f}\left(s, \frac{a}{q}\right)\left(\psi^{\prime}\left(\frac{k+1}{2}-s\right)-\psi^{\prime}\left(s+\frac{k-1}{2}\right)\right),
$$

so $I_{L}(z)=I_{L 1}(z)+I_{L 2}(z)$, where

$$
\begin{aligned}
& I_{L 1}(z)=\frac{1}{2 \pi i} \int_{\Re(s)=-\varepsilon} \epsilon \xi(q)\left(N q^{2}\right)^{\frac{1}{2}-s} \Delta_{\bar{f},-\overline{N a}, q}(1-s)(-i z)^{-s-\frac{k-1}{2}} d s, \\
& I_{L 2}(z)=\frac{1}{2 \pi i} \int_{\Re(s)=-\varepsilon} \Lambda_{f}\left(s, \frac{a}{q}\right)\left(\psi^{\prime}\left(\frac{k+1}{2}-s\right)-\psi^{\prime}\left(s+\frac{k-1}{2}\right)\right)(-i z)^{-s-\frac{k-1}{2}} d s .
\end{aligned}
$$


Making the substitution $s \mapsto 1-s$ in (2.10), we get

$$
\begin{aligned}
I_{L 1}(z) & =\frac{1}{2 \pi i} \int_{\Re(s)=1+\varepsilon} \epsilon \xi(q)\left(N q^{2}\right)^{s-\frac{1}{2}} \Delta_{\bar{f},-\overline{N a}, q}(s)(-i z)^{s-\frac{k+1}{2}} d s \\
& =2 \epsilon \xi(q)\left(N q^{2}\right)^{-\frac{1}{2}}(-i z)^{-\frac{k+1}{2}}(2 \pi)^{-\frac{k-1}{2}} \frac{1}{2 \pi i} \int_{\Re(s)=1+\varepsilon} \Delta_{\bar{f},-\overline{N a}, q}(s)\left(\frac{2 \pi}{-i N q^{2} z}\right)^{-s} d s \\
& =2 \epsilon \xi(q)\left(N q^{2}\right)^{-\frac{1}{2}}(-i z)^{-\frac{k+1}{2}}(2 \pi)^{-\frac{k-1}{2}} \sum_{n=1}^{\infty} c_{\bar{f},-\overline{N a}, q}(n)\left(\frac{2 \pi n}{-i N q^{2} z}\right)^{\frac{k-1}{2}} e\left(-\frac{n}{N q^{2} z}\right) \\
& =\frac{2 \epsilon \xi(q)}{(-i \sqrt{N} q z)^{k}} \sum_{n=1}^{\infty} c_{\bar{f},-\overline{N a}, q}(n) n^{\frac{k-1}{2}} e\left(-\frac{n}{N q^{2} z}\right) \\
& =\frac{\epsilon \xi(q)}{(-i \sqrt{N} q z)^{k}} \bar{F}\left(-\frac{1}{N q^{2} z}\right) .
\end{aligned}
$$

Next, note that the integrand in (2.11) is holomorphic for $-\frac{k-1}{2}<\Re(s)<\frac{k+1}{2}$. Moving the contour to $\Re(s)=\frac{k}{2}$, we get a contribution from the pole at $s=0$ (present only when $k=1$ ) of

$$
\operatorname{Res}_{s=0} \Lambda_{f}\left(s, \frac{a}{q}\right) \psi^{\prime}\left(s+\frac{k-1}{2}\right)(-i z)^{-s-\frac{k-1}{2}}=-\operatorname{Res}_{s=0} \Delta_{f, a, q}\left(s, \frac{a}{q}\right)(-i z)^{-s-\frac{k-1}{2}} .
$$

Thus

$$
\begin{aligned}
I_{L 2}(z)+\operatorname{Res}_{s=0} \Delta_{f, a, q}\left(s, \frac{a}{q}\right)(-i z)^{-s-\frac{k-1}{2}} & \\
& =\frac{1}{2 \pi i} \int_{\Re(s)=\frac{k}{2}} \Lambda_{f}\left(s, \frac{a}{q}\right)\left(\psi^{\prime}\left(\frac{k+1}{2}-s\right)-\psi^{\prime}\left(s+\frac{k-1}{2}\right)\right)(-i z)^{-s-\frac{k-1}{2}} d s .
\end{aligned}
$$

The reflection formula for $\Gamma$ implies that $\psi^{\prime}(1-s)+\psi^{\prime}(s)=\frac{\pi^{2}}{\sin ^{2}(\pi s)}$, so

$$
\psi^{\prime}\left(\frac{k+1}{2}-s\right)-\psi^{\prime}\left(s+\frac{k-1}{2}\right)=\frac{\pi^{2}}{\sin ^{2}\left(\pi\left(s+\frac{k-1}{2}\right)\right)}-\psi^{\prime}\left(s+\frac{k-1}{2}\right)-\psi^{\prime}\left(s-\frac{k-1}{2}\right) .
$$

Therefore $I_{L 2}(z)+\operatorname{Res}_{s=0} \Delta_{f, a, q}\left(s, \frac{a}{q}\right)(-i z)^{-s-\frac{k-1}{2}}=I_{L 2 B}(z)-I_{L 2 A}(z)$, where

$$
\begin{aligned}
& I_{L 2 A}(z)=\frac{1}{2 \pi i} \int_{\Re(s)=\frac{k}{2}} \Lambda_{f}\left(s, \frac{a}{q}\right)\left(\psi^{\prime}\left(s+\frac{k-1}{2}\right)+\psi^{\prime}\left(s-\frac{k-1}{2}\right)\right)(-i z)^{-s-\frac{k-1}{2}} d s, \\
& I_{L 2 B}(z)=\frac{1}{2 \pi i} \int_{\Re(s)=\frac{k}{2}} \Lambda_{f}\left(s, \frac{a}{q}\right) \frac{\pi^{2}}{\sin ^{2}\left(\pi\left(s+\frac{k-1}{2}\right)\right)}(-i z)^{-s-\frac{k-1}{2}} d s .
\end{aligned}
$$

Hence

$$
S_{f, a, q}(y, \alpha)=I_{R}(z)-I_{L 1}(z)+I_{L 2 A}(z)-I_{L 2 B}(z) .
$$

By applying (2.9), (2.12) and by setting $A(z)=I_{L 2 A}(z)$ and $B(z)=I_{L 2 B}(z)$, we establish Lemma 2.3.

Next we evaluate $\int_{0}^{|\alpha| / 4} S_{f, a, q}(y, \alpha) y^{s+\frac{k-1}{2}} \frac{d y}{y}$, considering each term on the right-hand side of (2.8) in turn.

Lemma 2.4. $\int_{0}^{|\alpha| / 4} F(\alpha+i y) y^{s+\frac{k-1}{2}} \frac{d y}{y}-\Delta_{f, a, q}(s, \alpha)$ continues to an entire function of $s$.

Proof. From the definition of $F$ we compute that

$$
\int_{0}^{\infty} F(\alpha+i y) y^{s+\frac{k-1}{2}} \frac{d y}{y}=\Delta_{f, a, q}(s, \alpha) .
$$


Moreover, $F(\alpha+i y)$ decays exponentially as $y \rightarrow \infty$, so the contribution to the integral from $y>|\alpha| / 4$ is entire.

Lemma 2.5. For any $M \in \mathbb{Z}_{\geq 0}$,

$$
\begin{aligned}
& \int_{0}^{|\alpha| / 4}(-i \sqrt{N} q(\alpha+i y))^{-k} \bar{F}\left(-\frac{1}{N q^{2}(\alpha+i y)}\right) y^{s+\frac{k-1}{2}} \frac{d y}{y} \\
& -(i \operatorname{sgn} \alpha)^{k} \sum_{m=0}^{M-1}(-i \alpha)^{-m}\left(\begin{array}{c}
s+m-\frac{k+1}{2} \\
m
\end{array}\right)\left(N q^{2} \alpha^{2}\right)^{s-\frac{1}{2}+m} \Delta_{\bar{f},-\overline{N a}, q}\left(s+m,-\frac{1}{N q^{2} \alpha}\right)
\end{aligned}
$$

continues to a holomorphic function on $\{s \in \mathbb{C}: \Re(s)>1-M\}$.

Proof. As the proof of this lemma is very similar to that of [Boo16, Lemma 3.3], we just provide a sketch and refer to the appropriate parts of loc. cit. for the relevant details. Fix $y \in(0,|\alpha| / 4]$, and set $z=\alpha+i y, \beta=-1 / N q^{2} \alpha$, and $u=y / \alpha$. It may be checked that

$$
-\frac{1}{N q^{2} z}=\beta+i|\beta u|-\frac{\beta u^{2}}{1+i u}
$$

Therefore

$$
\begin{aligned}
& (-i \sqrt{N} q z)^{-k} \bar{F}\left(-\frac{1}{N q^{2} z}\right) \\
& =2(-i \sqrt{N} q \alpha)^{-k} \sum_{n=1}^{\infty} c_{\bar{f},-\overline{N a}, q}(n) n^{\frac{k-1}{2}} e(\beta n) e^{-2 \pi n|\beta u|}(1+i u)^{-k} e\left(-\frac{n \beta u^{2}}{1+i u}\right) .
\end{aligned}
$$

It was shown in [Boo16, p. 820] that

$$
(1+i u)^{-k} e\left(-\frac{n \beta u^{2}}{1+i u}\right)=\sum_{m=0}^{\infty}(-i u)^{m} \sum_{j=0}^{m}\left(\begin{array}{c}
m+k-1 \\
m-j
\end{array}\right) \frac{(-2 \pi n|\beta u|)^{j}}{j !}
$$

and for $M, K \in \mathbb{Z}_{\geq 0}$ and $|u| \leq \frac{1}{4}$,

$$
\sum_{m=M}^{\infty}(-i u)^{m} \sum_{j=0}^{m}\left(\begin{array}{c}
m+k-1 \\
m-j
\end{array}\right) \frac{(-2 \pi n|\beta u|)^{j}}{j !} \ll_{\alpha, M, K}|u|^{M-K} n^{-K} e^{2 \pi n|\beta u|}
$$

Thus, we obtain

$$
\begin{aligned}
& (-i \sqrt{N} q z)^{-k} \bar{F}\left(-\frac{1}{N q^{2} z}\right)=O_{M, K}\left(y^{M-K} \sum_{n=1}^{\infty}\left|c_{\bar{f},-\overline{N a}, q}(n)\right| n^{\frac{k-1}{2}-K}\right)+2(-i \sqrt{N} q \alpha)^{-k} \\
& \times \sum_{m=0}^{M-1}\left(-\frac{i y}{\alpha}\right)^{m} \sum_{j=0}^{m}\left(\begin{array}{c}
m+k-1 \\
m-j
\end{array}\right) \sum_{n=1}^{\infty} c_{\bar{f},-\overline{N a}, q}(n) n^{\frac{k-1}{2}} e(\beta n) \frac{1}{j !}\left(-\frac{2 \pi n y}{N q^{2} \alpha^{2}}\right)^{j} e^{-\frac{2 \pi n y}{N q^{2} \alpha^{2}}} .
\end{aligned}
$$


By the choice $K=\left\lfloor\frac{k-1}{2}\right\rfloor+2$, the error term converges and is $O_{M}\left(y^{M-K}\right)$. For the other term note that

$$
\begin{aligned}
& 2 y^{m} \sum_{n=1}^{\infty} c_{\bar{f},-\overline{N a}, q}(n) n^{\frac{k-1}{2}} e(\beta n) \frac{1}{j !}\left(-\frac{2 \pi n y}{N q^{2} \alpha^{2}}\right)^{j} e^{-\frac{2 \pi n y}{N q^{2} \alpha^{2}}} \\
& =2 \frac{y^{j+m}}{j !} \frac{d^{j}}{d y^{j}} \sum_{n=1}^{\infty} c_{\bar{f},-\overline{N a}, q}(n) n^{\frac{k-1}{2}} e(\beta n) e^{-\frac{2 \pi n y}{N q^{2} \alpha^{2}}} \\
& =\frac{y^{j+m}}{j !} \frac{d^{j}}{d y^{j}} \frac{1}{2 \pi i} \int_{\Re(s)=m+2}\left(N q^{2} \alpha^{2}\right)^{s+\frac{k-1}{2}} \Delta_{\bar{f},-\overline{N a}, q}(s, \beta) y^{-s-\frac{k-1}{2}} d s \\
& =\frac{1}{2 \pi i} \int_{\Re(s)=2}\left(\begin{array}{c}
-s-\frac{k-1}{2}-m \\
j
\end{array}\right)\left(N q^{2} \alpha^{2}\right)^{s+\frac{k-1}{2}+m} \Delta_{\bar{f},-\overline{N a}, q}(s+m, \beta) y^{-s-\frac{k-1}{2}} d s .
\end{aligned}
$$

Inserting this in the last term of (2.14) and using the Chu-Vandermonde identity

$$
\sum_{j=0}^{m}\left(\begin{array}{c}
m+k-1 \\
m-j
\end{array}\right)\left(\begin{array}{c}
-s-\frac{k-1}{2}-m \\
j
\end{array}\right)=\left(\begin{array}{c}
-s+\frac{k-1}{2} \\
m
\end{array}\right)=(-1)^{m}\left(\begin{array}{c}
s+m-\frac{k+1}{2} \\
m
\end{array}\right),
$$

we arrive at

$$
\begin{aligned}
& (-i \sqrt{N} q z)^{-k} \bar{F}\left(-\frac{1}{N q^{2} z}\right) \\
& =O_{M}\left(y^{M-\left\lfloor\frac{k+3}{2}\right\rfloor}\right)+(i \operatorname{sgn} \alpha)^{k} \sum_{m=0}^{M-1} \frac{(-i \alpha)^{-m}}{2 \pi i} \int_{\Re(s)=2}\left(\begin{array}{c}
s+m-\frac{k+1}{2} \\
m
\end{array}\right)\left(N q^{2} \alpha^{2}\right)^{s-\frac{1}{2}+m} \\
& \cdot \Delta_{\bar{f},-\overline{N a}, q}\left(s+m,-\frac{1}{N q^{2} \alpha}\right) y^{-s-\frac{k-1}{2}} d s .
\end{aligned}
$$

We multiply both sides by $y^{s+\frac{k-1}{2}-1}$ and integrate over $y \in(0,|\alpha| / 4]$. The error term yields a holomorphic function for $\Re(s)>2-M$. As for the sum over $m$, by shifting the contour to the right, we see that each term decays rapidly as $y \rightarrow \infty$, so the integral over $(0,|\alpha| / 4]$ differs from the full Mellin transform by an entire function. By Mellin inversion, it follows that (2.13) is holomorphic for $\Re(s)>2-M$. Finally, replacing $M$ by $M+1$ and discarding the final term of the sum concludes the proof of the lemma.

Lemma 2.6. $\Gamma_{\mathbb{C}}(s)^{-1} \int_{0}^{|\alpha| / 4} A(\alpha+i y) y^{s} \frac{d y}{y}$ continues to an entire function of $s$.

Proof. Let $\Phi(s)=\psi^{\prime}\left(s+\frac{k-1}{2}\right)+\psi^{\prime}\left(s-\frac{k-1}{2}\right)$. By the identity $\psi^{\prime}(s)=\int_{1}^{\infty} \frac{\log x}{x-1} x^{-s} d x$, we have $\Phi(s)=\int_{1}^{\infty} \phi(x) x^{-s-\frac{k-1}{2}} d x$ for $\Re(s)>\frac{k-1}{2}$, where $\phi(x)=\frac{x^{k-1}+1}{x-1} \log x$. It follows that

$\Phi(s) \Gamma\left(s+\frac{k-1}{2}\right)=\int_{1}^{\infty} \phi(x) x^{-s-\frac{k-1}{2}} d x \int_{0}^{\infty} e^{-y} y^{s+\frac{k-1}{2}} d y=\int_{1}^{\infty} \phi(x) \int_{0}^{\infty} e^{-y}\left(\frac{y}{x}\right)^{s+\frac{k-1}{2}} \frac{d y}{y}$.

By the variable change $y \mapsto x y$ we obtain

$$
\Phi(s) \Gamma\left(s+\frac{k-1}{2}\right)=\int_{1}^{\infty} \phi(x) \int_{0}^{\infty} e^{-x y} y^{s+\frac{k-1}{2}} \frac{d y}{y}=\int_{0}^{\infty}\left(\int_{1}^{\infty} \phi(x) e^{-x y} d x\right) y^{s+\frac{k-1}{2}} \frac{d y}{y} .
$$

By Mellin inversion,

$$
\int_{1}^{\infty} \phi(x) e^{-x y} d x=\frac{1}{2 \pi i} \int_{\substack{\Re(s)=2 \\ 9}} \Phi(s) \Gamma\left(s+\frac{k-1}{2}\right) y^{-s-\frac{k-1}{2}} d s .
$$


Observe that $L_{\bar{f}}\left(s,-\frac{\overline{N a}}{q}\right)=\sum_{n=1}^{\infty} b_{n} n^{-s}$, where $b_{n}=\lambda_{\bar{f}}(n) e\left(-\frac{\overline{N a}}{q}\right)$. Thus for $z \in \mathbb{H}$,

$$
\begin{aligned}
A(z) & =2 \sum_{n=1}^{\infty} b_{n} \cdot \frac{1}{2 \pi i} \int_{\Re(s)=2} \Phi(s) \Gamma\left(s+\frac{k-1}{2}\right)(-2 \pi i n z)^{-s-\frac{k-1}{2}} d s \\
& =2 \sum_{n=1}^{\infty} b_{n} \int_{1}^{\infty} \phi(x) e^{2 \pi i n x z} d x
\end{aligned}
$$

where the last step follows from (2.15). For $z=\alpha+i y$ this simplifies to

$$
A(\alpha+i y)=2 \sum_{n=1}^{\infty} b_{n} \int_{1}^{\infty} \phi(x) e(\alpha n x) e^{-2 \pi n x y} d x .
$$

Using this expression, it follows that

$$
\begin{aligned}
& \int_{0}^{\infty} A(\alpha+i y) y^{s} \frac{d y}{y}=2 \sum_{n=1}^{\infty} b_{n} \int_{1}^{\infty} \phi(x) e(\alpha n x) \int_{0}^{\infty} e^{-2 \pi n x y} y^{s} \frac{d y}{y} d x \\
& =\Gamma_{\mathbb{C}}(s) \sum_{n=1}^{\infty} b_{n} n^{-s} \int_{1}^{\infty} \phi(x) e(\alpha n x) x^{-s} d x .
\end{aligned}
$$

For $j=0,1,2, \ldots$, define the sequence of functions $\phi_{j}(x, s)$ by

$$
\phi_{0}(x, s)=\phi(x), \quad \phi_{j+1}(x, s)=x \frac{\partial \phi_{j}}{\partial x}(x, s)-(s+j) \phi_{j}(x, s) .
$$

Integrating by parts,

$$
\int_{1}^{\infty} \phi_{j}(x, s) e(\alpha n x) x^{-s-j} d x=-\frac{e(\alpha n) \phi_{j}(1, s)}{2 \pi i \alpha n}-\frac{1}{2 \pi i \alpha n} \int_{1}^{\infty} \phi_{j+1}(x, s) e(\alpha n x) x^{-s-j-1} d x .
$$

Repeated application of this yields

$$
\begin{aligned}
\int_{1}^{\infty} \phi(x) e(\alpha n x) x^{-s} d x & =e(\alpha n) \sum_{j=0}^{m-1} \frac{\phi_{j}(1, s)}{(-2 \pi i \alpha n)^{j+1}} \\
& +(-2 \pi i \alpha n)^{-m} \int_{1}^{\infty} \phi_{m}(x, s) e(\alpha n x) x^{-s-m} d x
\end{aligned}
$$

for $m \in \mathbb{Z}_{\geq 0}$. By (2.17) and (2.18) it follows that

$$
\begin{aligned}
\frac{1}{\Gamma_{\mathbb{C}}(s)} \int_{0}^{\infty} A(\alpha+i y) y^{s} \frac{d y}{y} & =\sum_{j=0}^{m-1} \frac{\phi_{j}(1, s)}{(-2 \pi i \alpha)^{j+1}} L_{\bar{f}}\left(s+j+1,-\frac{\overline{N a}}{q}+\alpha\right) \\
& +(-2 \pi i \alpha)^{-m} \sum_{n=1}^{\infty} \frac{b_{n}}{n^{s+m}} \int_{1}^{\infty} \phi_{m}(x, s) e(\alpha n x) x^{-s-m} d x .
\end{aligned}
$$

Each term in the sum extends to an entire function of $s$, by [BK11, Proposition 3.1]. Furthermore, it may be checked that $\phi_{m}(x, s) \ll_{m, k}(1+|s|)^{m} x^{k-1}$. Therefore the last integral is holomorphic for $\Re(s)>k-m$. Letting $m \rightarrow \infty$ shows that $\Gamma_{\mathbb{C}}(s)^{-1} \int_{0}^{\infty} A(\alpha+$ iy) $y^{s} \frac{d y}{y}$ continues to an entire function.

Finally, from (2.16) we see that $A(\alpha+i y)$ decays exponentially as $y \rightarrow \infty$, and hence $\int_{|\alpha| / 4}^{\infty} A(\alpha+i y) y^{s} \frac{d y}{y}$ is entire. This completes the proof.

Lemma 2.7. $\Gamma_{\mathbb{C}}(s)^{-1} \int_{0}^{|\alpha| / 4} B(\alpha+i y) y^{s} \frac{d y}{y}$ continues to an entire function of $s$. 
Proof. Following the proof of [Boo16, Lemma 3.4], we obtain

$$
B(\alpha+i y)=\sum_{j=0}^{M-1} P_{j}(\alpha) y^{j}+O_{M}\left(y^{M}\right) \quad \text { for all } M \in \mathbb{Z}_{\geq 0}, y \in\left(0, \frac{|\alpha|}{4}\right],
$$

where

$P_{j}(\alpha)=\frac{(-i \alpha)^{-j}}{2 \pi i} \int_{\Re(s)=\frac{k}{2}} e^{i \frac{\pi}{2} \operatorname{sgn}(\alpha)\left(s+\frac{k-1}{2}\right)}|\alpha|^{-s-\frac{k-1}{2}}\left(\begin{array}{c}-s-\frac{k-1}{2} \\ j\end{array}\right) \Lambda_{f}\left(s, \frac{a}{q}\right) \frac{\pi^{2}}{\sin ^{2}\left(\pi\left(s+\frac{k-1}{2}\right)\right)} d s$.

Hence,

$$
\int_{0}^{|\alpha| / 4} B(\alpha+i y) y^{s} \frac{d y}{y}-\sum_{j=0}^{M-1} P_{j}(\alpha) \frac{|\alpha / 4|^{s+j}}{s+j}
$$

is holomorphic for $\Re(s)>-M$. Note that the sum over $j$ is entire apart from at most simple poles at the poles of $\Gamma_{\mathbb{C}}(s)$. Dividing by $\Gamma_{\mathbb{C}}(s)$ and taking $M \rightarrow \infty$ concludes the proof.

Proof of Proposition 2.2. Combining Lemmas 2.3 2.7 and taking $M=1$, we see that $I_{f, a, q, \alpha}(s)-H_{f, a, q, \alpha}(s)$ has analytic continuation to $\Re(s)>0$. If $\int_{0}^{|\alpha| / 4}\left|S_{f, a, q}(y, \alpha)\right| y^{\sigma+\frac{k-1}{2}} \frac{d y}{y}<$ $\infty$ for some $\sigma \geq 0$, then the integral defining $I_{f, a, q, \alpha}(s)$ converges absolutely for $\Re(s)>\sigma$, and hence $I_{f, a, q, \alpha}(s)$ is holomorphic in that region.

\section{Estimates FOR $N_{f, a, q}^{s}(T)$}

Fix $f, a, q$ as in Proposition 2.1, and let $\alpha \in \mathbb{Q}^{\times}$. In this section, we derive estimates for $N_{f, a, q}^{s}(T)$ based on Proposition 2.2.

Lemma 3.1. Let $f \in S_{k}\left(\Gamma_{1}(N)\right)$ be a primitive form. For $\rho=\beta+i \gamma$ a zero of $\Lambda_{f}(s)$, we have

$$
\Lambda_{f}^{\prime}(\rho) \ll_{f}(2+|\gamma|)^{\frac{k}{2}+\frac{\left|\beta-\frac{1}{2}\right|}{3}-\frac{1}{6}} \log ^{2}(2+|\gamma|) e^{-\frac{\pi}{2}|\gamma|} .
$$

Proof. We begin by establishing, for $s=\sigma+i t$ and $\sigma \in\left[\frac{1}{2}, 1\right]$,

$$
\Gamma_{\mathbb{C}}\left(s+\frac{k-1}{2}\right) L_{f}^{\prime}(s) \ll \tau^{\frac{k}{2}-\frac{1-\sigma}{3}} e^{-\frac{\pi}{2}|t|} \log ^{2} \tau,
$$

where $\tau=|t|+2$. By [BMN19, Theorem 1.1], we have

$$
L_{f}\left(\frac{1}{2}+i t\right) \ll \tau^{\frac{1}{3}} \log \tau .
$$

By the Phragmén-Lindelöf principle, using

$$
L_{f}\left(-\frac{1}{\log \tau}+i t\right) \ll \tau \log \tau \quad \text { and } \quad L_{f}\left(1+\frac{1}{\log \tau}+i t\right) \ll \log \tau,
$$

it follows that $L_{f}(\sigma+i t) \ll \tau^{\frac{1}{3}} \log \tau$ when $\left|\sigma-\frac{1}{2}\right| \leq 1 / \log \tau$. An application of the Cauchy integral formula then yields $L_{f}^{\prime}\left(\frac{1}{2}+i t\right) \ll \tau^{\frac{1}{3}} \log ^{2} \tau$. By Cauchy's inequality and Rankin's estimate $\sum_{n \leq x}\left|\lambda_{f}(n)\right|^{2} \ll x$, we get

$$
\left|L_{f}^{\prime}(1+\varepsilon+i t)\right| \leq \sum_{n=1}^{\infty} \frac{\left|\lambda_{f}(n)\right| \log n}{n^{1+\varepsilon}} \leq\left(\sum_{n=1}^{\infty} \frac{\left|\lambda_{f}(n)\right|^{2}}{n^{1+\varepsilon}}\right)^{\frac{1}{2}} \zeta^{\prime \prime}(1+\varepsilon)^{\frac{1}{2}} \ll \varepsilon^{-\frac{1}{2}} \varepsilon^{-\frac{3}{2}}=\varepsilon^{-2}
$$

for $\varepsilon>0$. Another application of the Phragmén-Lindelöf principle yields

$$
L_{f}^{\prime}(\sigma+i t) \ll \tau^{\frac{2}{3}(1-\sigma)} \log ^{2} \tau
$$


for $\sigma \in\left[\frac{1}{2}, 1\right]$. This, together with the Stirling formula estimate

$$
\Gamma_{\mathbb{C}}\left(s+\frac{k-1}{2}\right) \ll \tau^{\sigma+\frac{k}{2}-1} e^{-\frac{\pi}{2}|t|},
$$

yields (3.2).

Setting $s=\rho=\beta+i \gamma$ with $\beta \geq \frac{1}{2}$ in (3.2) gives

$$
\Lambda_{f}^{\prime}(\rho)=\Gamma_{\mathbb{C}}\left(\rho+\frac{k-1}{2}\right) L_{f}^{\prime}(\rho) \ll(2+|\gamma|)^{\frac{k}{2}-\frac{1-\beta}{3}} \log ^{2}(2+|\gamma|) e^{-\frac{\pi}{2}|\gamma|} .
$$

Now suppose $\beta<\frac{1}{2}$. Differentiating the functional equation we obtain

$$
\Lambda_{f}^{\prime}(\rho)=-\epsilon N^{\frac{1}{2}-\rho} \Lambda_{\bar{f}}^{\prime}(1-\rho),
$$

where $|\epsilon|=1$. Applying (3.3) to $\Lambda_{\bar{f}}^{\prime}(1-\rho)$ it follows that

$$
\Lambda_{f}^{\prime}(\rho) \ll(2+|\gamma|)^{\frac{k}{2}-\frac{\beta}{3}} \log ^{2}(2+|\gamma|) e^{-\frac{\pi}{2}|\gamma|} .
$$

Combining (3.3) and (3.4) we obtain (3.1).

Lemma 3.2. For any fixed $\varepsilon>0$ and all $\sigma \in[\varepsilon, 2]$,

$$
\int_{0}^{\frac{|\alpha|}{4}}\left|S_{f, a, q}(y, \alpha)\right| y^{\sigma+\frac{k-1}{2}} \frac{d y}{y} \ll \sum_{\substack{\rho=\beta+i \gamma \\ a \text { pole of } \Delta_{f, a, q}^{*}(s)}}(2+|\gamma|)^{\frac{1+\left|\beta-\frac{1}{2}\right|}{3}-\sigma} \log ^{2}(2+|\gamma|) .
$$

Proof. Throughout this proof we let $\rho=\beta+i \gamma$ denote a pole of $\Delta_{f, a, q}^{*}(s)$, and we set $\tau=2+|\gamma|$. Recalling (2.6) $)$, observe that $(y-i \alpha)^{-\rho-\frac{k-1}{2}}=e^{i \frac{\pi}{2} \operatorname{sgn}(\alpha)\left(\rho+\frac{k-1}{2}\right)}|\alpha|^{-\rho-\frac{k-1}{2}}(1+$ $\left.\frac{i y}{\alpha}\right)^{-\rho-\frac{k-1}{2}}$ and

$$
\begin{aligned}
\left|\left(1+i \frac{y}{\alpha}\right)^{-\left(\beta+i \gamma+\frac{k-1}{2}\right)}\right| & =\left|e^{-\left(\frac{1}{2} \log \left(1+(y / \alpha)^{2}\right)+i \arctan (y / \alpha)\right)\left(\beta+i \gamma+\frac{k-1}{2}\right)}\right| \\
& =\left(1+\left(\frac{y}{\alpha}\right)^{2}\right)^{-\frac{\beta}{2}-\frac{k-1}{4}} e^{\gamma \arctan (y / \alpha)} .
\end{aligned}
$$

Therefore

$$
(y-i \alpha)^{-\rho-\frac{k-1}{2}} \ll e^{\gamma \operatorname{sgn}(\alpha)\left(\arctan (y /|\alpha|)-\frac{\pi}{2}\right)} .
$$

Next, we treat the residue in (2.6). By (2.4), the poles of $\Delta_{f, a, q}^{*}(s)$ arise from poles of $\Delta_{f}(s)$ and $\Delta_{f}(s, \chi)$ with $\chi \neq \chi_{0}$. The contributrion of an individual term of (2.4) to $\operatorname{Res}_{s=\rho} \Delta_{f, a, q}^{*}(s)$, if nonzero, is of the form

$$
-\left(1-\frac{q}{q-1} P_{f, q}\left(q^{-\rho}\right)\right) \Lambda_{f}^{\prime}(\rho) \quad \text { or } \quad-\frac{\tau(\bar{\chi}) \chi(a)}{q-1} \Lambda_{f}^{\prime}(\rho, \chi) .
$$

Applying Lemma 3.1 (possibly replacing $f$ by $f \otimes \chi$ ) to each of these expressions, it follows that

$$
\operatorname{Res}_{s=\rho} \Delta_{f, a, q}^{*}(s) \ll \tau^{\frac{k}{2}+\frac{\left|\beta-\frac{1}{2}\right|}{3}-\frac{1}{6}}\left(\log ^{2} \tau\right) e^{-\frac{\pi}{2}|\gamma|} .
$$

It follows from (2.6), (3.6), and (3.7) that

$$
S_{f, a, q}(y, \alpha) \ll \sum_{\rho} \tau^{\frac{k}{2}+\frac{\left|\beta-\frac{1}{2}\right|}{3}-\frac{1}{6}}\left(\log ^{2} \tau\right) e^{|\gamma|\left[\operatorname{sgn}(\alpha \gamma) \arctan (y /|\alpha|)-\frac{\pi}{2}(1+\operatorname{sgn}(\alpha \gamma))\right]} .
$$

By considering cases and using the bound $\arctan u \geq \frac{u}{2}$ for $0 \leq u \leq \frac{1}{4}$, we have

$$
S_{f, a, q}(y, \alpha) \ll \sum_{\rho} \tau^{\frac{k}{2}+\frac{\left|\beta-\frac{1}{2}\right|}{3}-\frac{1}{6}}\left(\log ^{2} \tau\right) e^{-c|\gamma| y} \quad \text { for } y \in\left(0, \frac{|\alpha|}{4}\right],
$$


where $c=\frac{1}{2|\alpha|}>0$. We deduce from this

$$
\int_{0}^{\frac{|\alpha|}{4}}\left|S_{f, a, q}(y, \alpha)\right| y^{\sigma+\frac{k-1}{2}} \frac{d y}{y} \ll \sum_{\rho} \tau^{\frac{k}{2}+\frac{\left|\beta-\frac{1}{2}\right|}{3}-\frac{1}{6}} \log ^{2} \tau \int_{0}^{\frac{|\alpha|}{4}} e^{-c|\gamma| y} y^{\sigma+\frac{k-1}{2}} \frac{d y}{y} .
$$

Now

$$
\int_{0}^{\frac{|\alpha|}{4}} e^{-c|\gamma| y} y^{\sigma+\frac{k-1}{2}} \frac{d y}{y} \ll \int_{0}^{\frac{|\alpha|}{4}} e^{-c \tau y} y^{\sigma+\frac{k-1}{2}} \frac{d y}{y} \leq \int_{0}^{\infty} e^{-c \tau y} y^{\sigma+\frac{k-1}{2}} \frac{d y}{y} .
$$

By the variable change $u=c \tau y$, the last integral equals

$$
\frac{1}{(c \tau)^{\sigma+\frac{k-1}{2}}} \int_{0}^{\infty} e^{-u} u^{\sigma+\frac{k-1}{2}} \frac{d u}{u}=\frac{\Gamma\left(\sigma+\frac{k-1}{2}\right)}{(c \tau)^{\sigma+\frac{k-1}{2}}} \ll \tau^{-\sigma-\frac{k-1}{2}},
$$

and thus

$$
\int_{0}^{\frac{|\alpha|}{4}} S_{f, a, q}(y, \alpha) y^{\sigma+\frac{k-1}{2}} \frac{d y}{y} \ll \sum_{\rho} \tau^{\frac{1+\left|\beta-\frac{1}{2}\right|}{3}-\sigma} \log ^{2} \tau
$$

For a meromorphic function $h$ on $\{s \in \mathbb{C}: \Re(s)>1\}$, define

$$
\Theta(h)=\inf \{\theta \geq 0: h \text { continues analytically to }\{s \in \mathbb{C}: \Re(s)>\theta\}\} .
$$

We also set

$$
\theta_{f, a, q}(T)=\sup \left(\{0\} \cup\left\{\Re(\rho), 1-\Re(\rho): \rho \in \mathbb{C},|\Im(\rho)| \leq T, \operatorname{Res}_{s=\rho} \Delta_{f, a, q}^{*}(s) \neq 0\right\}\right)
$$

and

By Proposition 2.1, we have

$$
\theta_{f, a, q}=\lim _{T \rightarrow \infty} \theta_{f, a, q}(T)
$$

$$
\theta_{f, a, q}=\max \left(\Theta\left(\Delta_{f, a, q}\right), \Theta\left(\Delta_{\bar{f},-\overline{N a}, q}\right)\right) .
$$

Proposition 3.3. If $\Theta\left(H_{f, a, q, \alpha}\right)>0$ then $\theta_{f, a, q} \geq \frac{1}{2}$ and

$$
N_{f, a, q}^{s}(T)=\Omega\left(T^{\frac{1}{3}\left(1-\theta_{f, a, q}\right)+\Theta\left(H_{f, a, q, \alpha}\right)-\frac{1}{2}-\varepsilon}\right) \quad \text { for all } \varepsilon>0 .
$$

Further, if $\Theta\left(H_{f, a, q, \alpha}\right)=\frac{1}{2}$ and $H_{f, a, q, \alpha}(s)$ has a pole with real part $\frac{1}{2}$, then

$$
N_{f, a, q}^{s}(T)=\Omega\left(\frac{T^{\frac{1}{3}\left(1-\theta_{f, a, q}(T)\right)}}{\left(1-\theta_{f, a, q}(T)\right) \log ^{2} T}\right)
$$

and there are arbitrarily large $T>0$ such that

$$
N_{f, a, q}^{s}(T) \geq \log \log \log T \text {. }
$$

Proof. Let $\beta_{n}+i \gamma_{n}$ run through the poles of $\Delta_{f, a, q}^{*}(s)$, in increasing order of $\left|\gamma_{n}\right|$. For brevity, we write $I(s), H(s), \Theta, S(y), N(t), \theta(t)$ and $\theta$ for $I_{f, a, q, \alpha}(s), H_{f, a, q, \alpha}(s), \Theta\left(H_{f, a, q, \alpha}\right)$, $S_{f, a, q}(y, \alpha), N_{f, a, q}^{s}(t), \theta_{f, a, q}(t)$ and $\theta_{f, a, q}$, respectively. By Lemma 3.2, we have

$$
\begin{aligned}
\int_{0}^{|\alpha| / 4}|S(y)| y^{\sigma+\frac{k-1}{2}} \frac{d y}{y} & \ll \sum_{n \geq 1}\left(2+\left|\gamma_{n}\right|\right)^{\frac{1+\left|\beta_{n}-\frac{1}{2}\right|}{3}-\sigma} \log ^{2}\left(2+\left|\gamma_{n}\right|\right) \\
& \leq \sum_{n \geq 1}\left(2+\left|\gamma_{n}\right|\right)^{\frac{\theta\left(\left|\gamma_{n}\right|\right)}{3}+\frac{1}{6}-\sigma} \log ^{2}\left(2+\left|\gamma_{n}\right|\right) .
\end{aligned}
$$


If $\Theta>0$ then by Proposition 2.2, the integral must diverge for sufficiently small $\sigma>0$, and thus the right-hand side has infinitely many terms. Thus $\Delta_{f, a, q}^{*}(s)$ has poles, so $\theta \geq \frac{1}{2}$.

Suppose that (3.9) does not hold. Then there exists $\varepsilon \in(0, \Theta)$ such that $N(t)=$ $o\left(t^{\frac{1}{3}(1-\theta)+\Theta-\frac{1}{2}-\varepsilon}\right)$. Choosing $\sigma=\Theta-\frac{\varepsilon}{3}$ and using the estimate $\log ^{2}\left(2+\left|\gamma_{n}\right|\right) \ll\left(2+\left|\gamma_{n}\right|\right)^{\frac{\varepsilon}{3}}$, we have

$$
\begin{aligned}
\int_{0}^{|\alpha| / 4}|S(y)| y^{\Theta-\frac{\varepsilon}{3}+\frac{k-1}{2}} \frac{d y}{y} & \ll \sum_{n \geq 1}\left(2+\left|\gamma_{n}\right|\right)^{\frac{\theta}{3}+\frac{1}{6}-\Theta+\frac{2}{3} \varepsilon} \ll 1+\int_{1}^{\infty} t^{\frac{\theta}{3}+\frac{1}{6}-\Theta+\frac{2}{3} \varepsilon} d N(t) \\
& \ll 1+\int_{1}^{\infty} t^{\frac{\theta}{3}+\frac{1}{6}-\Theta+\frac{2}{3} \varepsilon-1} N(t) d t \ll 1+\int_{1}^{\infty} t^{-1-\frac{\varepsilon}{3}} d t \ll 1 .
\end{aligned}
$$

By Proposition 2.2, it follows that $H(s)$ is holomorphic for $\Re(s)>\Theta-\frac{\varepsilon}{3}$. This is a contradiction, so (3.9) must hold.

Next suppose that $\Theta=\frac{1}{2}$, and let $\rho$ be a pole of $H(s)$ with $\Re(\rho)=\frac{1}{2}$. Then for sufficiently small $\delta>0$, by Proposition 2.2, we have

$$
\delta^{-1} \ll|H(\rho+\delta)| \ll 1+|I(\rho+\delta)| \leq 1+\int_{0}^{|\alpha| / 4}|S(y)| y^{\delta+\frac{k}{2}} \frac{d y}{y},
$$

where we understand the right-hand side to be $\infty$ if the integral diverges. Applying Lemma 3.2, we thus have

$$
\delta^{-1} \ll 1+\sum_{n \geq 1}\left(2+\left|\gamma_{n}\right|\right)^{\frac{\theta\left(\left|\gamma_{n}\right|\right)-1}{3}-\delta} \log ^{2}\left(2+\left|\gamma_{n}\right|\right) .
$$

In particular, the right-hand side must have infinitely many terms. Applying integration by parts, we get

$$
\begin{aligned}
\delta^{-1} & \ll 1+\int_{1}^{\infty} t^{\frac{\theta(t)-1}{3}-\delta} \log ^{2} t d N(t)=1-\int_{1}^{\infty} N(t) d\left(t^{\frac{\theta(t)-1}{3}-\delta} \log ^{2} t\right) \\
& \leq 1+\int_{1}^{\infty} N(t)\left(\frac{1-\theta(t)}{3}+\delta\right) t^{\frac{\theta(t)-1}{3}-\delta-1} \log ^{2} t d t
\end{aligned}
$$

where for the last inequality we have used the fact that $\theta(t)$ is nondecreasing and

$$
d\left(t^{\frac{\theta(t)-1}{3}-\delta} \log ^{2} t\right)=t^{\frac{\theta(t)-1}{3}-\delta-1}(\log t)\left[2-\left(\frac{1-\theta(t)}{3}+\delta\right) \log t\right] d t+\frac{1}{3} \log ^{3} t d \theta(t) .
$$

Suppose that (3.10) is false, so that the function $\varepsilon(t)=N(t) t^{\frac{1}{3}(\theta(t)-1)}(1-\theta(t)) \log ^{2} t$ satisfies $\lim _{t \rightarrow \infty} \varepsilon(t)=0$. Then we have

$$
\delta^{-1} \ll 1+\int_{1}^{\infty}\left(\frac{1}{3}+\frac{\delta}{1-\theta(t)}\right) \varepsilon(t) t^{-1-\delta} d t .
$$

By the standard zero-free region [IK04, Theorem 5.10], we have

$$
\frac{1}{1-\theta(t)} \ll \log \max (t, 2)
$$

so that

$$
\delta^{-1} \ll 1+\int_{1}^{\infty}(1+\delta \log t) \varepsilon(t) t^{-1-\delta} d t=1+\delta^{-1} \int_{0}^{\infty} \varepsilon\left(e^{u / \delta}\right)(1+u) e^{-u} d u=o\left(\delta^{-1}\right) .
$$

This is a contradiction, so (3.10) holds. 
Finally, suppose (3.11) is false, so that $N(T)<\log \log \log T$ for all sufficiently large $T$. Then there exists $n_{0} \geq \mathbb{Z}_{>0}$ such that $\left|\gamma_{n}\right|>\exp \exp \exp n$ for all $n \geq n_{0}$. Since the terms from $n<n_{0}$ contribute a bounded amount to (3.12), we have

$$
1+\sum_{n=n_{0}}^{\infty}\left|\gamma_{n}\right|^{-\delta} \log ^{2}\left|\gamma_{n}\right| \gg \delta^{-1}
$$

for all sufficiently small $\delta>0$.

Next we claim that there are infinitely many $m \geq n_{0}$ such that

$$
\log \log \left|\gamma_{m+1}\right| \geq \frac{13}{5} \log \log \left|\gamma_{m}\right| \text {. }
$$

If not then there exists $n_{1} \geq n_{0}$ such that (3.13) fails for all $m \geq n_{1}$, and by induction it follows that

$$
\log \log \left|\gamma_{n}\right| \leq\left(\frac{13}{5}\right)^{n-n_{1}} \log \log \left|\gamma_{n_{1}}\right|=c\left(\frac{13}{5}\right)^{n} \quad \text { for } n \geq n_{1},
$$

where $c=(13 / 5)^{-n_{1}} \log \log \left|\gamma_{n_{1}}\right|>0$. Hence,

$$
n<\log \log \log \left|\gamma_{n}\right| \leq \log c+n \log \frac{13}{5} .
$$

Since $\log \frac{13}{5}<1$, this is false for sufficiently large $n$, proving the claim.

Choose a large $m \geq n_{0}$ satisfying (3.13), and set $\delta_{m}=\left(\log \left|\gamma_{m}\right|\right)^{-\frac{12}{5}}$. Then using the trivial bound $e^{e^{e}} \leq\left|\gamma_{n}\right| \leq\left|\gamma_{m}\right|$ for $n_{0} \leq n \leq m$, we have

$$
\sum_{n=n_{0}}^{m}\left|\gamma_{n}\right|^{-\delta_{m}} \log ^{2}\left|\gamma_{n}\right| \leq m \log ^{2}\left|\gamma_{m}\right|<\left(\log \log \log \left|\gamma_{m}\right|\right) \log ^{2}\left|\gamma_{m}\right| \leq\left(\log \left|\gamma_{m}\right|\right)^{\frac{11}{5}}=\delta_{m}^{-\frac{11}{12}}
$$

since $\log \log x \leq x^{\frac{1}{5}}$ for all $x>1$.

To estimate the contribution from $n>m$ we apply integration by parts. Set $g(t)=$ $t^{-\delta_{m}} \log ^{2} t$. Then $g^{\prime}(t)<0$ for $t>e^{2 / \delta_{m}}=\exp \left(2\left(\log \left|\gamma_{m}\right|\right)^{12 / 5}\right)$; in particular, if $m$ is sufficiently large then, by (3.13),$g^{\prime}(t)<0$ for $t \geq\left|\gamma_{m+1}\right|$. Hence, we have

$$
\begin{aligned}
\sum_{n=m+1}^{\infty} g\left(\left|\gamma_{n}\right|\right) & =\lim _{\varepsilon \rightarrow 0^{+}} \int_{\left|\gamma_{m+1}\right|-\varepsilon}^{\infty} g(t) d N(t)=\int_{\left|\gamma_{m+1}\right|}^{\infty}\left(-g^{\prime}(t)\right)(N(t)-m) d t \\
& \leq \int_{\left|\gamma_{m+1}\right|}^{\infty}\left(-g^{\prime}(t)\right)(\log \log \log t) d t \leq \delta_{m} \int_{\left|\gamma_{m+1}\right|}^{\infty} t^{-\delta_{m}-1}(\log t)^{\frac{11}{5}} d t \\
& =\delta_{m} \int_{\log \left|\gamma_{m+1}\right|}^{\infty} e^{-\delta_{m} u} u^{\frac{11}{5}} d u
\end{aligned}
$$

Applying integration by parts three times and using that $\delta_{m} \log \left|\gamma_{m+1}\right| \gg 1$, we get

$$
\delta_{m} \int_{\log \left|\gamma_{m+1}\right|}^{\infty} e^{-\delta_{m} u} u^{\frac{11}{5}} d u \ll\left|\gamma_{m+1}\right|^{-\delta_{m}}\left(\log \left|\gamma_{m+1}\right|\right)^{\frac{11}{5}} .
$$

Note that $\delta_{m}=\left(\log \left|\gamma_{m}\right|\right)^{-\frac{12}{5}} \geq\left(\log \left|\gamma_{m+1}\right|\right)^{-\frac{12}{13}}$, so $\left|\gamma_{m+1}\right|^{-\delta_{m}} \leq \exp \left(-\left(\log \left|\gamma_{m+1}\right|\right)^{\frac{1}{13}}\right)$. Hence, we conclude that

$$
\sum_{n=m+1}^{\infty} g\left(\left|\gamma_{n}\right|\right) \ll \exp \left(-\left(\log \left|\gamma_{m+1}\right|\right)^{\frac{1}{13}}\right)\left(\log \left|\gamma_{m+1}\right|\right)^{\frac{11}{5}} \ll 1
$$

Thus, altogether we have

$$
\delta_{m}^{-1} \ll 1+\sum_{n=n_{0}}^{\infty}\left|\gamma_{n}\right|^{-\delta_{m}} \log ^{2}\left|\gamma_{n}\right| \ll 1+\delta_{m}^{-\frac{11}{12}} .
$$

This is false for sufficiently large $m$, so (3.11) must hold for some arbitrarily large $T$. 


\section{Proofs of Theorems 1.1 and 1.2}

We begin with an overview of the argument. By Proposition $3.3, N_{f, a, q}^{s}(T)$ is sometimes large if there exists $\alpha \in \mathbb{Q}^{\times}$for which $H_{f, a, q, \alpha}(s)$ has a pole with large real part. The main obstacle to showing this is that $H_{f, a, q, \alpha}(s)$ is defined as the difference of two functions (cf. (2.7) ), whose poles could in principle cancel out. However, as we show, there are some dependencies between $H_{f, a, q, \alpha}(s)$ for various choices of $(a, q, \alpha)$, from which it follows that there is a suitable pole for at least one choice of inputs. More specifically, in Lemma 4.1 we exhibit a relationship between $H_{f, 1,1, a / p}(s)$ and $H_{f, a, q,-a / q}(s)$, where $p$ and $q$ are primes satisfying $p q \equiv-1(\bmod N a)$. For any prime $p \nmid N$, we show that there is some choice of $a \in \mathbb{Z}$ for which this leads to poles at the simple zeros of $\Lambda_{f}(s)$, and thanks to Boo16, Theorem 1.1], those exist in abundance. Ultimately this implies that at least one of $N_{f}^{s}(T), N_{f, a, p}^{s}(T), N_{f, a, q}^{s}(T)$ is large, which yields Theorem 1.1. Choosing $p=2$ and appealing to the second and third conclusions of Proposition 3.3 yields Theorem 1.2 .

Proceeding, given a prime $p$ and $a \in \mathbb{Z}$ coprime to $p$, define

$$
C_{f, a, p}(s)=\Delta_{f, a, p}(s)-\xi(p) p^{1-2 s} \Delta_{f}(s) .
$$

Lemma 4.1. Let $a \in \mathbb{Z}$, and let $p$ and $q$ be prime numbers such that $p q \equiv-1(\bmod N a)$. Then

(i) $C_{f, a, p}(s)-\left(H_{f, 1,1, a / p}(s)-\xi(p) p^{1-2 s} H_{f, a, q,-a / q}(s)\right)$ is holomorphic for $\Re(s)>0$;

(ii) $\sum_{b=1}^{p-1} C_{f, b, p}(s)=-P_{f, p}\left(p^{1-s}\right) \Delta_{f}(s)$.

Proof. We first consider $H_{f, a, q, \alpha}(s)$, where $\alpha=-a / q$. We have

$$
\Delta_{f, a, q}(s, \alpha)-\Delta_{f}(s)=-R_{f, q}\left(q^{-s}\right) \Lambda_{f}(s),
$$

which is holomorphic for $\Re(s)>0$. Set $a^{\prime}=-\frac{1+p q}{N a}$, so that $\frac{a^{\prime}}{q}-\frac{1}{N q^{2} \alpha}=-\frac{p}{N a}$. Let $r_{\bar{f}, q}(j)$ be the numbers such that

$$
R_{\bar{f}, q}(x)=\sum_{j=1}^{\infty} r_{\bar{f}, q}(j) x^{j}
$$

By Fourier inversion, we have

$$
\sum_{\substack{j \geq 1 \\ j \equiv t(\bmod \varphi(N a))}} r_{\bar{f}, q}(j) x^{j}=\frac{1}{\varphi(N a)} \sum_{\ell=1}^{\varphi(N a)} e\left(-\frac{\ell t}{\varphi(N a)}\right) R_{\bar{f}, q}\left(e\left(\frac{\ell}{\varphi(N a)}\right) x\right) .
$$

Thus,

$$
\begin{aligned}
\Delta_{\bar{f}, a^{\prime}, q} & \left(s,-\frac{1}{N q^{2} \alpha}\right)-\Delta_{\bar{f}}\left(s,-\frac{p}{N a}\right)=-\sum_{j=1}^{\infty} r_{\bar{f}, q}(j) q^{-j s} \Lambda_{\bar{f}}\left(s, \frac{q^{j-1}}{N a}\right) \\
= & -\sum_{t=1}^{\varphi(N a)} \Lambda_{\bar{f}}\left(s, \frac{q^{t-1}}{N a}\right) \sum_{\substack{j \geq 1 \\
j \equiv t(\bmod \varphi(N a))}} r_{\bar{f}, q}(j) q^{-j s} \\
= & -\frac{1}{\varphi(N a)} \sum_{t=1}^{\varphi(N a)} \Lambda_{\bar{f}}\left(s, \frac{q^{t-1}}{N a}\right) \sum_{\ell=1}^{\varphi(N a)} e\left(-\frac{\ell t}{\varphi(N a)}\right) R_{\bar{f}, q}\left(e\left(\frac{\ell}{\varphi(N a)}\right) q^{-s}\right),
\end{aligned}
$$

which is again holomorphic for $\Re(s)>0$. Hence, up to a holomorphic function, $H_{f, a, q, \alpha}(s)$ is

$$
\Delta_{f}(s)-\epsilon \xi(q)(-i \operatorname{sgn} a)^{k}\left(N a^{2}\right)^{s-\frac{1}{2}} \Delta_{\bar{f}}\left(s,-\frac{p}{N a}\right) .
$$


Next note that

$C_{f, a, p}(s)-H_{f, 1,1, a / p}(s)=\epsilon(i \operatorname{sgn} a)^{k}\left(\frac{N a^{2}}{p^{2}}\right)^{s-\frac{1}{2}} \Delta_{\bar{f}}\left(s,-\frac{p}{N a}\right)-\xi(p) p^{1-2 s} \Delta_{f}(s)-R_{f, q}\left(q^{-s}\right) \Lambda_{f}(s)$.

Therefore, since $\xi(p) \xi(q)=\xi(-1)=(-1)^{k}$, we see that

$$
C_{f, a, p}(s)-H_{f, 1,1, a / p}(s)+\xi(p) p^{1-2 s} H_{f, a, q, \alpha}(s)
$$

is holomorphic for $\Re(s)>0$.

Finally, by (2.4) we have

$$
\begin{aligned}
\sum_{b=1}^{p-1} C_{f, b, p}(s) & =(p-1)\left[1-\frac{p}{p-1} P_{f, p}\left(p^{-s}\right)-\xi(p) p^{1-2 s}\right] \Delta_{f}(s) \\
& =-P_{f, p}\left(p^{1-s}\right) \Delta_{f}(s) .
\end{aligned}
$$

In the following we shall make frequent use of the observation that for any pair $h_{1}, h_{2}$ of meromorphic functions,

$$
\Theta\left(h_{1}+h_{2}\right) \leq \max \left(\Theta\left(h_{1}\right), \Theta\left(h_{2}\right)\right), \quad \text { with equality when } \Theta\left(h_{1}\right) \neq \Theta\left(h_{2}\right) .
$$

Fix a prime $p \nmid N$. By [Boo16, Theorem 1.1] and the functional equation, $\Delta_{f}(s)$ has a pole with real part $\geq \frac{1}{2}$, and thus

$$
\Theta\left(\Delta_{f}\right)=\theta_{f, 1,1} \geq \frac{1}{2}
$$

Since all zeros of $P_{f, p}\left(p^{1-s}\right)$ have real part 1, this is also true of $P_{f, p}\left(p^{1-s}\right) \Delta_{f}(s)$. Hence, by Lemma 4.1(ii), there exists $a \in\{1, \ldots, p-1\}$ such that $C_{f, a, p}(s)$ has a pole with real part $\geq \frac{1}{2}$ and satisfies $\Theta\left(C_{f, a, p}\right) \geq \theta_{f, 1,1}$. By (4.1) and (4.2), it follows that

$$
\Theta\left(C_{f, a, p}\right)=\max \left(\Theta\left(\Delta_{f, a, p}\right), \theta_{f, 1,1}\right) \text {. }
$$

Let $q$ be a prime satisfying $p q \equiv-1(\bmod N a)$, and set $a^{\prime}=-(1+p q) /(N a)$.

We aim to prove that

$$
\max \left(N_{f}^{s}(T), N_{f, a, p}^{s}(T), N_{f, a, q}^{s}(T)\right)=\Omega\left(T^{\frac{1}{6}-\varepsilon}\right) \quad \text { for all } \varepsilon>0 .
$$

To that end, we will show that at least one of the following inequalities holds for some $\alpha \in \mathbb{Q}^{\times}$:

(i) $\max \left(\Theta\left(H_{f, 1,1, \alpha}\right), \Theta\left(H_{\bar{f}, 1,1, \alpha}\right)\right) \geq \theta_{f, 1,1} \geq \frac{1}{2}$;

(ii) $\max \left(\Theta\left(H_{f, a, p, \alpha}\right), \Theta\left(H_{\bar{f}, a^{\prime}, p, \alpha}\right)\right) \geq \theta_{f, a, p} \geq \frac{1}{2}$;

(iii) $\max \left(\Theta\left(H_{f, a, q, \alpha}\right), \Theta\left(H_{\bar{f}, a^{\prime}, q, \alpha}\right)\right) \geq \theta_{f, a, q} \geq \frac{1}{2}$.

To see that this suffices, suppose for instance that (iii) holds. By Proposition 2.1, we have $N_{f, a, q}^{s}(T)=N_{\bar{f}, a^{\prime}, q}^{s}(T)$ and $\theta_{f, a, q}=\theta_{\bar{f}, a^{\prime}, q}$. Thus, applying Proposition 3.3 to either $(f, a, q)$ or $\left(\bar{f}, a^{\prime}, q\right)$, we conclude that

$$
N_{f, a, q}^{s}(T)=\Omega\left(T^{\beta-\varepsilon}\right), \quad \text { where } \beta \geq \frac{1}{3}\left(1-\theta_{f, a, q}\right)+\theta_{f, a, q}-\frac{1}{2}=\frac{2 \theta_{f, a, q}}{3}-\frac{1}{6} \geq \frac{1}{6} .
$$

If, instead, (i) or (ii) holds, then by a similar argument we find that $N_{f, 1,1}^{s}(T)=\Omega\left(T^{\beta-\varepsilon}\right)$ or $N_{f, a, p}^{s}(T)=\Omega\left(T^{\beta-\varepsilon}\right)$ for some $\beta \geq \frac{1}{6}$. Hence, (4.5) follows in any case.

Let us suppose that conditions (i) and (iii) are false for all $\alpha \in \mathbb{Q}^{\times}$and show that this leads to (ii). Since (i) is false, in view of (4.3) we must have $\theta_{f, 1,1}>\Theta\left(H_{f, 1,1, a / p}\right)$. In turn, by (4.4) this implies that $\Theta\left(C_{f, a, p}\right)>\Theta\left(H_{f, 1,1, a / p}\right)$. Hence, by Lemma 4.1(i) and (4.2), 
we have $\Theta\left(H_{f, a, q,-a / q}\right)=\Theta\left(C_{f, a, p}\right)$. By (4.4), this implies $\Theta\left(H_{f, a, q,-a / q}\right) \geq \theta_{f, 1,1}>0$, and thus $\theta_{f, a, q} \geq \frac{1}{2}$, by Proposition 3.3 .

Next, by (3.8) we have $\theta_{f, a, p}=\max \left(\Theta\left(\Delta_{f, a, p}\right), \Theta\left(\Delta_{\bar{f}, a^{\prime}, p}\right)\right)$. If

$$
\Theta\left(\Delta_{\bar{f}, a^{\prime}, p}\right) \leq \max \left(\Theta\left(\Delta_{f, a, p}\right), \theta_{f, 1,1}\right)=\Theta\left(H_{f, a, q,-a / q}\right)
$$

then it follows that

$$
\Theta\left(H_{f, a, q,-a / q}\right)=\max \left(\theta_{f, a, p}, \theta_{f, 1,1}\right) .
$$

Suppose now that (4.6) is false. Then $\Theta\left(\Delta_{\bar{f}, a^{\prime}, p}\right)>\max \left(\Theta\left(\Delta_{f, a, p}\right), \theta_{f, 1,1}\right)$, so that

$$
\theta_{f, a, p}=\Theta\left(\Delta_{\bar{f}, a^{\prime}, p}\right)>\theta_{f, 1,1} .
$$

Since (i) is false, this implies that $\Theta\left(\Delta_{\bar{f}, a^{\prime}, p}\right)>\max \left(\Theta\left(H_{\bar{f}, 1,1, a^{\prime} / p}\right), \theta_{f, 1,1}\right)$. By (4.1) and Lemma 4.1(i) with $\left(\bar{f}, a^{\prime}\right)$ in place of $(f, a)$, it follows from (4.2) that

$$
\Theta\left(H_{\bar{f}, a^{\prime}, q,-a^{\prime} / q}\right)=\Theta\left(\Delta_{\bar{f}, a^{\prime}, p}\right)=\max \left(\theta_{f, a, p}, \theta_{f, 1,1}\right) .
$$

Therefore, since at least one of (4.7) and (4.8) must hold, we have

$$
\max \left(\Theta\left(H_{f, a, q,-a / q}\right), \Theta\left(H_{\bar{f}, a^{\prime}, q,-a^{\prime} / q}\right)\right) \geq \max \left(\theta_{f, a, p}, \theta_{f, 1,1}\right) .
$$

Since (iii) is false, this implies that $\theta_{f, a, q}>\max \left(\theta_{f, a, p}, \theta_{f, 1,1}\right)$. Hence, by (3.8), either

$$
\Theta\left(\Delta_{f, a, q}\right)>\max \left(\theta_{f, a, p}, \theta_{f, 1,1}\right) \quad \text { or } \quad \Theta\left(\Delta_{\bar{f}, a^{\prime}, q}\right)>\max \left(\theta_{f, a, p}, \theta_{f, 1,1}\right) .
$$

Suppose that the first inequality in (4.9) holds. Then by (4.1) (with $q$ in place of $p$ ) and (4.2), we have $\Theta\left(C_{f, a, q}\right)=\Theta\left(\Delta_{f, a, q}\right)>\theta_{f, 1,1}$. Since (i) is false, this implies $\Theta\left(C_{f, a, q}\right)>\Theta\left(H_{f, 1,1, a / q}\right)$. On the other hand, by Lemma 4.1(i) (with the roles of $p$ and $q$ reversed) and (4.2), we have

$$
\Theta\left(H_{f, a, p,-a / p}\right)=\Theta\left(C_{f, a, q}\right)=\Theta\left(\Delta_{f, a, q}\right)>\theta_{f, a, p} .
$$

This also implies that $\Theta\left(H_{f, a, p,-a / p}\right)>0$, whence $\theta_{f, a, p} \geq \frac{1}{2}$, by Proposition 3.3 .

If, instead, the second inequality holds in (4.9), then running through the same argument with $\left(\bar{f}, a^{\prime}\right)$ in place of $(f, a)$, we find that

$$
\Theta\left(H_{\bar{f}, a^{\prime}, p,-a^{\prime} / p}\right)=\Theta\left(C_{\bar{f}, a^{\prime}, q}\right)=\Theta\left(\Delta_{\bar{f}, a^{\prime}, q}\right)>\theta_{\bar{f}, a^{\prime}, p} \geq \frac{1}{2} .
$$

Hence, in either case we see that (ii) holds, and this concludes the proof of (4.5).

Now, by (4.5) and (2.4), it follows that there is a character $\chi$ of conductor $1, p$ or $q$ such that $N_{f \otimes \chi}^{s}(T)=\Omega\left(T^{\frac{1}{6}-\varepsilon}\right)$ for all $\varepsilon>0$. This implies Theorem 1.1.

For the proof of Theorem 1.2, we may assume that $N_{f}^{s}(T) \ll 1+T^{\varepsilon}$ for all $\varepsilon>0$, since the result is trivial otherwise. To avoid contradicting Proposition 3.3, it must therefore be the case that $\max \left(\Theta\left(H_{f, 1,1, \alpha}\right), \Theta\left(H_{\bar{f}, 1,1, \alpha}\right)\right) \leq \frac{1}{2}$ for all $\alpha \in \mathbb{Q}^{\times}$.

Since $N$ is odd, we can take $p=2$ and $a=1$ in the above, and choose any suitable prime $q$. Then by Lemma 4.1(ii), we have

$$
\Delta_{f, a, p}(s)=\left(\xi(p) p^{1-2 s}-P_{f, p}\left(p^{1-s}\right)\right) \Delta_{f}(s),
$$

and it follows that $N_{f, a, p}^{s}(T) \leq N_{f}^{s}(T)$ and $\max \left(\Theta\left(H_{f, a, p, \alpha}\right), \Theta\left(H_{\bar{f}, a^{\prime}, p, \alpha}\right)\right) \leq \frac{1}{2}$ for all $\alpha \in \mathbb{Q}^{\times}$. Thus, by (4.5),$N_{f, a, q}^{s}(T)=\Omega\left(T^{\frac{1}{6}-\varepsilon}\right)$ for all $\varepsilon>0$. Therefore, by Proposition 2.1, at least one of $\Delta_{f, a, q}(s), \Delta_{\bar{f}, a^{\prime}, q}(s)$ has a pole in the region $\left\{s \in \mathbb{C}: \Re(s) \geq \frac{1}{2}\right\}$ that is not a pole of $\Delta_{f}(s)$. By (4.1) and Lemma 4.1(i), the same applies to one of $H_{f, 1,1, a / q}(s)$, $H_{f, a, p,-a / p}(s), H_{\bar{f}, 1,1, a^{\prime} / q}(s)$, or $H_{\bar{f}, a^{\prime}, p,-a^{\prime} / p}(s)$.

Since

$$
N_{f, a, p}^{s}(T)=N_{\bar{f}, a^{\prime}, p}^{s}(T) \underset{18}{\leq} N_{f, 1,1}^{s}(T)=N_{\bar{f}, 1,1}^{s}(T),
$$


whichever function has the pole, we can apply Proposition 3.3 to see that $N_{f}^{s}(T)$ satisfies the second and third conclusions. In particular, $N_{f}^{s}(T) \geq \log \log \log T$ for some arbitrarily large $T$, and if $k=1$ or $f$ is a CM form then Coleman's theorem Col90] implies that

$$
1-\theta_{f, a, p}(T) \geq 1-\theta_{f, 1,1}(T) \gg(\log T)^{-\frac{2}{3}}(\log \log T)^{-\frac{1}{3}} \quad \text { for all } T \geq 3,
$$

whence $N_{f}^{s}(T)=\Omega\left(\exp \left((\log T)^{\frac{1}{3}-\varepsilon}\right)\right)$ for all $\varepsilon>0$. Moreover, since $N_{f}^{s}(T) \ll 1+T^{\varepsilon}$, we must have $\theta_{f, 1,1}=1$, so $\Lambda_{f}(s)$ has simple zeros with real part arbitrarily close to 1 .

Finally, by Lemma 4.1(ii) we have $\Theta\left(C_{f, a, p}\right)=1$. Since $\Theta\left(H_{f, 1,1, a / p}\right) \leq \frac{1}{2}$, Lemma 4.1(i) and (4.2) imply that $\Theta\left(H_{f, a, q,-a / q}\right)=1$. Applying Proposition [3.3, it follows that $N_{f, a, q}^{s}(T)=\Omega\left(T^{\frac{1}{2}-\varepsilon}\right)$ for all $\varepsilon>0$. By Lemma 4.1 $(\mathrm{i}), C_{f, a, q}(s)$ and $C_{\bar{f}, a^{\prime}, q}(s)$ are holomorphic for $\Re(s)>\frac{1}{2}$. Hence, by (4.1) and Proposition 2.1, all poles of $\Delta_{f, a, q}^{*}(s)$ that are not poles of $\Delta_{f}^{*}(s)$ lie on the line $\left\{s \in \mathbb{C}: \Re(s)=\frac{1}{2}\right\}$. Since $N_{f}^{s}(T) \ll 1+T^{\varepsilon}, \Delta_{f, a, q}^{*}(s)$ must have $\Omega\left(T^{\frac{1}{2}-\varepsilon}\right)$ poles with real part $\frac{1}{2}$ and imaginary part in $[-T, T]$. By (2.4), the same applies to $\Delta_{f}(s, \chi)$ for some $\chi(\bmod q)$.

\section{REFERENCES}

[BK11] Andrew R. Booker and M. Krishnamurthy, A strengthening of the GL(2) converse theorem, Compos. Math. 147 (2011), no. 3, 669-715. MR 2801397

[BMN19] Andrew R. Booker, Micah B. Milinovich, and Nathan Ng, Subconvexity for modular form L-functions in the $t$ aspect, Adv. Math. 341 (2019), 299-335. MR 3872849

[Boo16] Andrew R. Booker, Simple zeros of degree 2 L-functions, J. Eur. Math. Soc. (JEMS) 18 (2016), no. 4, 813-823. MR 3474457

[CG88] J. B. Conrey and A. Ghosh, Simple zeros of the Ramanujan $\tau$-Dirichlet series, Invent. Math. 94 (1988), no. 2, 403-419. MR 958837 (89k:11078)

[Col90] M. D. Coleman, A zero-free region for the Hecke L-functions, Mathematika 37 (1990), no. 2, 287-304. MR 1099777

[IK04] Henryk Iwaniec and Emmanuel Kowalski, Analytic number theory, American Mathematical Society Colloquium Publications, vol. 53, American Mathematical Society, Providence, RI, 2004. MR 2061214

[JS77] Hervé Jacquet and Joseph A. Shalika, A non-vanishing theorem for zeta functions of $\mathrm{GL}_{n}$, Invent. Math. 38 (1976/77), no. 1, 1-16. MR 0432596 (55 \#5583)

[KMV02] E. Kowalski, P. Michel, and J. VanderKam, Rankin-Selberg L-functions in the level aspect, Duke Math. J. 114 (2002), no. 1, 123-191. MR 1915038

[MN14] Micah B. Milinovich and Nathan Ng, Simple zeros of modular L-functions, Proc. Lond. Math. Soc. (3) 109 (2014), no. 6, 1465-1506. MR 3293156

[Mun18] Ritabrata Munshi, Sub-Weyl bounds for GL(2) L-functions, arXiv:1806.07352, 2018.

[Wei67] André Weil, Über die Bestimmung Dirichletscher Reihen durch Funktionalgleichungen, Math. Ann. 168 (1967), 149-156. MR 0207658 (34 \#7473)

School of Mathematics, University of Bristol, Bristol, BS8 1TW, UK

E-mail address: andrew.booker@bristol.ac.uk

Department of Mathematics, University of Mississippi, University, MS 38677 USA

E-mail address: mbmilino@olemiss.edu

Department of Mathematics and Computer Science, University of Lethbridge, LethBRIDGE, AB CANADA T1K 3M4

E-mail address: nathan.ng@uleth.ca 